\title{
Applications of Schauder's Fixed Point Theorem to Semipositone Singular Differential Equations
}

\author{
Zhongwei Cao, ${ }^{1}$ Chengjun Yuan, ${ }^{2}$ and Xiuling $\mathrm{Li}^{1}$ \\ ${ }^{1}$ Department of Applied Mathematics, Jilin University of Finance and Economics, Changchun, Jilin 130117, China \\ ${ }^{2}$ School of Mathematics and Computer, Harbin University, Harbin 150086, China \\ Correspondence should be addressed to Zhongwei Cao; caozw963@sina.com
}

Received 2 June 2014; Accepted 21 July 2014; Published 17 August 2014

Academic Editor: Daqing Jiang

Copyright (c) 2014 Zhongwei Cao et al. This is an open access article distributed under the Creative Commons Attribution License, which permits unrestricted use, distribution, and reproduction in any medium, provided the original work is properly cited.

We study the existence of positive periodic solutions of second-order singular differential equations. The proof relies on Schauder's fixed point theorem. Our results generalized and extended those results contained in the studies by Chu and Torres (2007) and Torres (2007) . In some suitable weak singularities, the existence of periodic solutions may help.

\section{Introduction}

The theory of second-order periodic differential equation has been widely studied [1-20] because of great practical importance. In this paper, we apply Schauder's fixed point theorem to study the existence of positive solutions of the second-order periodic differential equation

$$
x^{\prime \prime}+a(t) x=f(t, x)+e(t),
$$

where $a, e$ are continuous and 1-periodic functions and the nonlinearity $f$ is continuous in $(t, x), 1$-periodic in $t$, and singular at $x=0$. In addition, $e$ may take some negative values.

Beginning with the paper of Lazer and Solimini [13], the semilinear singular differential equation

$$
x^{\prime \prime}+a(t) x=\frac{p(t)}{x^{\lambda}}+e(t),
$$

where $a, p, e \in C[0,1]$ and $\lambda>0$, has received the attention of many researchers during the last two decades $[5,7,10,21]$. In [2], the author investigated the existence of positive solutions of (2) for three cases when $\widetilde{e}_{*}>0, \widetilde{e}_{*}=0$, and $\widetilde{e}^{*} \leq 0$ through a basic application of Schauder's fixed point theorem. Here, let $p^{*}$ and $p_{*}$ denote the essential supremum and infimum of a function $p \in L^{1}[0,1]$, if they exist. For any continuous function $p$ in $[0,1]$, we set

$$
\tilde{p}(t)=\int_{0}^{1} G(t, s) p(s) d s
$$

In [1], the authors generalized the results of [2] and considered the second-order periodic semilinear singular equation

$$
x^{\prime \prime}+a(t) x=\frac{p(t)}{x^{\alpha}}+q(t) x^{\beta}+e(t),
$$

with $a, p, q, e \in C[0,1]$ and $\alpha, \beta>0$. By Schauder's fixed point theorem, they established the existence of positive periodic solutions to (4), if $\widetilde{e}^{*}>0$ and $\widetilde{e}_{*}=0$.

Although [1, Theorem 3.1] can deal with the cases of the existence of positive periodic solutions to (4), the authors there did not consider these two cases as applications of the existence of positive periodic solutions of (4) if $\widetilde{e}^{*} \leq 0$ or $\widetilde{e}_{*}<0<\widetilde{e}^{*}$. We also generalize some of the results of $[1,2]$ in certain ways.

The remaining part of the paper is organized as follows. In Section 2, some preliminary results are given. In Sections 3 and 4 , we will state and prove the main results of the paper, as well as some applications to (2) and (4). In Section 5, we apply the results established in this paper to two specific equations. 


\section{Preliminaries}

Throughout this paper, we assume that the equation, known as Hill's equation,

$$
x^{\prime \prime}+a(t) x=0
$$

with periodic boundary conditions

$$
x(0)=x(1), \quad x^{\prime}(0)=x^{\prime}(1)
$$

satisfies the following standing hypothesis:

(A) associated Green's function $G(t, s)$ is nonnegative for all $(t, s) \in[0,1] \times[0,1]$.

In other words, the antimaximum principle holds. Under this assumption, the function $\widetilde{e}(t)$ is just the unique 1-periodic solution of the linear equation

$$
x^{\prime \prime}+a(t) x=e(t) .
$$

Now we make condition (A) clear. When $a(t)=k^{2}$, condition (A) is equivalent to $0<k^{2} \leq \mu_{1}=\pi^{2}$. Note that $\mu_{1}$ is the first eigenvalue of the linear problem with Dirichlet conditions $x(0)=0=x(1)$. For a nonconstant function $a(t)$, there is a $L^{p}$-criterion proved in [16]. Let $\mathbf{K}(q)$ denote the best Sobolev constant in the following inequality:

$$
C\|u\|_{q}^{2} \leq\left\|u^{\prime}\right\|_{2}^{2}, \quad \forall u \in H_{0}^{1}(0,1) .
$$

The explicit formula for $\mathbf{K}(q)$ is

$$
\mathbf{K}(q)=\left\{\begin{array}{cc}
\frac{2 \pi}{q}\left(\frac{2}{2+q}\right)^{1-2 / q}\left(\frac{\gamma(1 / q)}{\gamma((1 / 2)+(1 / q))}\right)^{2} \\
\text { if } 1 \leq q<\infty \\
\text { if } q=\infty,
\end{array}\right.
$$

where $\gamma$ is the gamma function.

We write $p>0$ if $p \geq 0$ for a.e. $t \in[0,1]$ and $p$ is positive in a set of positive measures.

Lemma 1 (see [16]). Assume that $a(t)>0$ and $a \in L^{p}[0,1]$ for some $1 \leq p \leq \infty$. If

$$
\|a\|_{p} \leq K\left(2 p^{*}\right)
$$

then the standing hypothesis (A) holds.

Remark 2. In $[3,12,16]$, the existence results are based on the positiveness of $G(t, s)>0$, which plays a very important role in employing some fixed point theorems in cones for completely continuous operators. Our assumption (A) only needs that $G(t, s)$ is nonnegative, and therefore our results cover the critical case, which was not covered in the above three papers.

\section{Main Results}

In this section, we establish the main result about the existence of positive periodic solutions for (1).
Theorem 3. Suppose that the following conditions hold.

$\left(\mathrm{H}_{1}\right)$ There exist nonnegative continuous functions $b, c, d$ on $[0,1]$ and $g, h$ on $(0,+\infty)$ such that $b \geq c>0, g>0$ is strictly decreasing, and $h / g$ is increasing. Moreover, there holds for any $t \in[0,1]$ and $x \in(0,+\infty)$

$$
c(t) g(x) \leq f(t, x) \leq b(t) g(x)+d(t) h(x) \text {. }
$$

$\left(\mathrm{H}_{2}\right)$ There exists a positive constant $R$ such that

$$
\begin{gathered}
R>g(R)\left(\widetilde{b}^{*}+\frac{h(R)}{g(R)} \widetilde{d}^{*}\right)+\widetilde{e}^{*}, \\
\widetilde{e}_{*} \geq g^{-1}\left(\frac{R-\widetilde{e}^{*}}{\widetilde{b}^{*}+(h(R) / g(R)) \widetilde{d}^{*}}\right)-g(R) \widetilde{c}_{*} .
\end{gathered}
$$

Then (1) has at least one positive periodic solution.

Proof. Let $C_{1}$ denote the set of all continuous 1-periodic functions. We define a completely continuous map $T: C_{1} \rightarrow$ $C_{1}$ by

$$
\begin{aligned}
(T x)(t) & :=\int_{0}^{1} G(t, s)[f(s, x(s))+e(s)] d s \\
& =\int_{0}^{1} G(t, s) f(s, x(s)) d s+\widetilde{e}(t) .
\end{aligned}
$$

It is clear that a periodic solution of (1) is just a fixed point of $T$. It suffices to prove the existence of the fixed point of map $T$.

Let $R$ be the positive constant in $\left(\mathrm{H}_{2}\right)$ and set

$$
r:=g^{-1}\left(\frac{R-\widetilde{e}^{*}}{\widetilde{b^{*}+(h(R) / g(R)) \widetilde{d}^{*}}}\right) .
$$

Combining (12) with the fact that $g$ is positive and strictly decreasing, we have $R>r>0$. We introduce a set by

$$
\Omega:=\left\{x \in C_{1}: r \leq x(t) \leq R, \forall t \in[0,1]\right\} .
$$

Obviously, $\Omega$ is a closed convex set. For any $x \in \Omega$ and $t \epsilon$ $[0,1]$, by assumption (11), we get

$$
(T x)(t) \geq \int_{0}^{1} G(t, s) c(s) g(x(s)) d s+\widetilde{e}(t) .
$$

Since $x(s) \leq R$ and $g$ is strictly decreasing, there holds

$$
(T x)(t) \geq g(R) \tilde{c}(t)+\widetilde{e}(t) \geq g(R) \widetilde{c}_{*}+\widetilde{e}_{*} .
$$

Together with assumption (13), the above inequality leads to

$$
(T x)(t) \geq g^{-1}\left(\frac{R-\widetilde{e}^{*}}{\widetilde{b}^{*}+(h(R) / g(R)) \widetilde{d}^{*}}\right)=r .
$$


Similar discussion shows that there holds, for any $x \in \Omega$ and $t \in[0,1]$,

$$
\begin{aligned}
& (T x)(t) \\
& \leq \int_{0}^{1} G(t, s) g(x(s))\left(b(s)+\frac{h(x(s))}{g(x(s))} d(s)\right) d s+\widetilde{e}(t) \\
& \quad \leq g(r)\left(\tilde{b}(t)+\frac{h(R)}{g(R)} \tilde{d}(t)\right)+\widetilde{e}(t) \\
& \quad \leq g(r)\left(\tilde{b}_{*}+\frac{h(R)}{g(R)} \tilde{d}_{*}\right)+\widetilde{e}_{*}=R .
\end{aligned}
$$

Consequently, we get the conclusion that there holds $T(\Omega) \subset$ $\Omega$. By Schauder's fixed point theorem, we obtain that there exists at least one fixed point of $T$, which completes the proof.

Applying Theorem 3 to a special case, we have the following result.

Corollary 4. Suppose that $\widetilde{e}^{*} \leq 0$ and there hold condition $\left(H_{1}\right)$ and the following one.

$\left(\mathrm{H}_{2}^{*}\right)$ There exists a positive constant $R$ such that

$$
\begin{gathered}
R>g(R)\left(\tilde{b}^{*}+\frac{h(R)}{g(R)} \tilde{d}^{*}\right), \\
\widetilde{e}_{*} \geq g^{-1}\left(\frac{R}{\widetilde{b}^{*}+(h(R) / g(R)) \widetilde{d}^{*}}\right)-g(R) \widetilde{c}_{*} .
\end{gathered}
$$

Then (1) has at least one positive periodic solution.

\section{Applications}

4.1. The Case $\widetilde{e}^{*} \leq 0$. In this subsection, we consider (4) in the cases when $\widetilde{e}^{*} \leq 0$. To meet the condition in Corollary 4 , we let

$$
g(x)=x^{-\alpha}, \quad h(x)=x^{\beta}, \quad x \in(0,+\infty) .
$$

We note that to verify the existence of the positive periodic solution of (4) by Corollary 4 an important step is to find $R>$ 0 such that

$$
\widetilde{e}_{*} \geq\left(\frac{\tilde{b}^{*}+\tilde{d}^{*} R^{\alpha+\beta}}{R}\right)^{1 / \alpha}-\frac{\widetilde{c}_{*}}{R^{\alpha}},
$$

where $b, c, d$ are the functions in condition $\left(\mathrm{H}_{1}\right)$. Let $m=1 / R$. Then finding $R>0$ with (23) is equivalent to finding $m>0$ satisfying

$$
\widetilde{e}_{*} \geq\left[m\left(\widetilde{b}^{*}+\widetilde{d}^{*}\left(\frac{1}{m}\right)^{\alpha+\beta}\right)\right]^{1 / \alpha}-\widetilde{c}_{*} m^{\alpha}
$$

To this end, we consider the following function:

$$
\begin{array}{r}
F(m):=\left[m\left(\widetilde{b}^{*}+\widetilde{d}^{*}\left(\frac{1}{m}\right)^{\alpha+\beta}\right)\right]^{1 / \alpha}-\widetilde{c}_{*} m^{\alpha} \\
=m^{\alpha}\left[\left(\widetilde{b}^{*} m^{1-\alpha^{2}}+\widetilde{d}^{*} m^{1-\alpha-\beta-\alpha^{2}}\right)^{1 / \alpha}-\widetilde{c}_{*}\right], \\
m \in(0,+\infty) .
\end{array}
$$

By direct calculation, we have

$$
\begin{aligned}
& F^{\prime}(m) \\
& =\alpha m^{\alpha-1}\left[\left(\widetilde{b}^{*} m^{1-\alpha^{2}}+\widetilde{d}^{*} m^{1-\alpha-\beta-\alpha^{2}}\right)^{1 / \alpha}-\widetilde{c}_{*}\right] \\
& +m^{\alpha}\left[\frac{1}{\alpha}\left(\widetilde{b}^{*} m^{1-\alpha^{2}}+\widetilde{d}^{*} m^{1-\alpha-\beta-\alpha^{2}}\right)^{(1 / \alpha)-1}\right. \\
& \times\left(\left(1-\alpha^{2}\right) \tilde{b}^{*} m^{-\alpha^{2}}\right. \\
& \left.\left.+\left(1-\alpha-\beta-\alpha^{2}\right) \tilde{d}^{*} m^{-\alpha-\beta-\alpha^{2}}\right)\right] \\
& =\frac{1}{\alpha} m^{\alpha-1} \\
& \times\left\{\alpha^{2}\left[\left(\widetilde{b}^{*} m^{1-\alpha^{2}}+\widetilde{d}^{*} m^{1-\alpha-\beta-\alpha^{2}}\right)^{1 / \alpha}-\widetilde{c}_{*}\right]\right. \\
& +m\left[\left(\widetilde{b}^{*} m^{1-\alpha^{2}}+\widetilde{d}^{*} m^{1-\alpha-\beta-\alpha^{2}}\right)^{(1 / \alpha)-1}\right. \\
& \times\left(\left(1-\alpha^{2}\right) \widetilde{b}^{*} m^{-\alpha^{2}}\right. \\
& +\left(1-\alpha-\beta-\alpha^{2}\right) \\
& \left.\left.\left.\times \widetilde{d}^{*} m^{-\alpha-\beta-\alpha^{2}}\right)\right]\right\} \\
& =\frac{1}{\alpha} m^{\alpha-1} \\
& \times\left\{\alpha^{2}\left[\left(\widetilde{b}^{*} m^{1-\alpha^{2}}+\bar{d}^{*} m^{1-\alpha-\beta-\alpha^{2}}\right)^{1 / \alpha}-\widetilde{c}_{*}\right]\right. \\
& +\left[\left(\widetilde{b}^{*} m^{1-\alpha^{2}}+\widetilde{d}^{*} m^{1-\alpha-\beta-\alpha^{2}}\right)^{(1 / \alpha)-1}\right. \\
& \times\left(\left(1-\alpha^{2}\right) \tilde{b}^{*} m^{1-\alpha^{2}}\right. \\
& \left.\left.\left.+\left(1-\alpha-\beta-\alpha^{2}\right) \bar{d}^{*} m^{1-\alpha-\beta-\alpha^{2}}\right)\right]\right\}
\end{aligned}
$$




$$
\begin{aligned}
& =\frac{1}{\alpha} m^{\alpha-1} \\
& \times\left\{\left[\alpha^{2}\left(\tilde{b}^{*} m^{1-\alpha^{2}}+\widetilde{d}^{*} m^{1-\alpha-\beta-\alpha^{2}}\right)^{1 / \alpha}\right.\right. \\
& +\left(\widetilde{b}^{*} m^{1-\alpha^{2}}+\widetilde{d}^{*} m^{1-\alpha-\beta-\alpha^{2}}\right)^{(1 / \alpha)-1} \\
& \times\left(\left(1-\alpha^{2}\right) \tilde{b}^{*} m^{1-\alpha^{2}}+\left(1-\alpha-\beta-\alpha^{2}\right)\right. \\
& \left.\left.\left.\times \widetilde{d}^{*} m^{1-\alpha-\beta-\alpha^{2}}\right)\right]-\alpha^{2} \widetilde{c}_{*}\right\} \\
& =\frac{1}{\alpha} m^{\alpha-1} \\
& \times\left\{\left(\widetilde{b}^{*} m^{1-\alpha^{2}}+\widetilde{d}^{*} m^{1-\alpha-\beta-\alpha^{2}}\right)^{(1 / \alpha)-1}\right. \\
& \times\left[\alpha^{2}\left(\widetilde{b}^{*} m^{1-\alpha^{2}}+\widetilde{d}^{*} m^{1-\alpha-\beta-\alpha^{2}}\right)\right. \\
& +\left(\left(1-\alpha^{2}\right) \tilde{b}^{*} m^{1-\alpha^{2}}+\left(1-\alpha-\beta-\alpha^{2}\right)\right. \\
& \left.\left.\left.\times \widetilde{d}^{*} m^{1-\alpha-\beta-\alpha^{2}}\right)\right]-\alpha^{2} \widetilde{c}_{*}\right\} \\
& =\frac{1}{\alpha} m^{\alpha-1} \\
& \times\left\{\left(\widetilde{b}^{*} m^{1-\alpha^{2}}+\widetilde{d}^{*} m^{1-\alpha-\beta-\alpha^{2}}\right)^{(1 / \alpha)-1}\right. \\
& \times\left[\tilde{b}^{*} m^{1-\alpha^{2}}+\left(\alpha^{2}+\left(1-\alpha-\beta-\alpha^{2}\right)\right)\right. \\
& \left.\left.\times \widetilde{d}^{*} m^{1-\alpha-\beta-\alpha^{2}}\right]-\alpha^{2} \widetilde{c}_{*}\right\} \\
& =\frac{1}{\alpha} m^{\alpha-1} \\
& \times\left\{\left(\widetilde{b}^{*} m^{1-\alpha^{2}}+\widetilde{d}^{*} m^{1-\alpha-\beta-\alpha^{2}}\right)^{(1 / \alpha)-1}\right. \\
& \times\left[\widetilde{b}^{*} m^{1-\alpha^{2}}+(1-\alpha-\beta) \widetilde{d}^{*} m^{1-\alpha-\beta-\alpha^{2}}\right] \\
& \left.-\alpha^{2} \widetilde{c}_{*}\right\} \text {. }
\end{aligned}
$$

Suppose that $1-\alpha^{2}>0$ and $1-\alpha-\beta-\alpha^{2}>0$. Set

$$
\begin{aligned}
\Phi(m)=: & \left(\tilde{b}^{*} m^{1-\alpha^{2}}+\widetilde{d}^{*} m^{1-\alpha-\beta-\alpha^{2}}\right)^{(1 / \alpha)-1} \\
& \times\left[\widetilde{b}^{*} m^{1-\alpha^{2}}+(1-\alpha-\beta) \widetilde{d}^{*} m^{1-\alpha-\beta-\alpha^{2}}\right], \\
& m \in[0,+\infty) .
\end{aligned}
$$

It is clear that $\Phi$ is continuous and increasing on $[0,+\infty)$. Since there hold $\Phi(0)=0$ and $\lim _{m \rightarrow+\infty} \Phi(m)=+\infty$, there exists a unique $m_{0} \in(0,+\infty)$ such that $\Phi\left(m_{0}\right)=\alpha^{2} \widetilde{c}_{*}$. It follows that $F^{\prime}\left(m_{0}\right)=0$. In addition, we note that $F^{\prime}(m)<0$ for $0<m<m_{0}$ and $F^{\prime}(m)>0$ for $m>m_{0}$. Hence, we get the conclusion that $F$ possesses a minimum at $m=m_{0}$. Based upon the above discussion, we have that $m_{0}$ is the best choice for satisfying inequality (24).

Theorem 5. Suppose that $0<\alpha, \beta<1,1-\alpha-\alpha^{2}-\beta>0$, and there exist nonnegative continuous functions $b, c, d$ on $[0,1]$ such that $b \geq c>0$ and

$$
\begin{gathered}
c(t) x^{-\alpha} \leq p(t) x^{-\alpha}+q(t) x^{\beta} \leq b(t) x^{-\alpha}+d(t) x^{\beta}, \\
t \in[0,1], x \in(0,+\infty) .
\end{gathered}
$$

If there holds

$$
0 \geq \widetilde{e}_{*} \geq m_{0}^{\alpha}\left[\left(\widetilde{b}^{*} m_{0}^{1-\alpha^{2}}+\widetilde{d}^{*} m_{0}^{1-\alpha-\beta-\alpha^{2}}\right)^{1 / \alpha}-\widetilde{c}_{*}\right]
$$

where $m_{0}$ is the unique positive solution of the equation

$$
\begin{aligned}
& \left(\widetilde{b}^{*} m^{1-\alpha^{2}}+\widetilde{d}^{*} m^{1-\alpha-\beta-\alpha^{2}}\right)^{(1 / \alpha)-1} \\
& \quad \times\left[\widetilde{b}^{*} m^{1-\alpha^{2}}+(1-\alpha-\beta) \widetilde{d}^{*} m^{1-\alpha-\beta-\alpha^{2}}\right]=\alpha^{2} \widetilde{c}_{*},
\end{aligned}
$$

then (4) has at least one positive periodic solution.

Proof. Let $g(x)=x^{-\alpha}, h(x)=x^{\beta}$, and $x \in(0,+\infty)$. It is obvious that $g>0$ is strictly decreasing and $h / g$ is nondecreasing. According to the assumption about $b, c, d$, we have that condition $\left(\mathrm{H}_{1}\right)$ of Theorem 3 holds.

Let $R=1 / m_{0}$. It follows from assumption (29) that there holds

$$
\widetilde{e}_{*} \geq g^{-1}\left(\frac{R}{\widetilde{b}^{*}+(h(R) / g(R)) \widetilde{d}^{*}}\right)-g(R) \widetilde{c}_{*} .
$$

It suffices to verify that $R$ satisfies

$$
R>g(R)\left(\widetilde{b}^{*}+\frac{h(R)}{g(R)} \widetilde{d}^{*}\right)
$$

which is equivalent to the inequality

$$
m_{0}^{\alpha+1}\left(\bar{b}^{*} m_{0}^{1-\alpha^{2}}+\bar{d}^{*} m_{0}^{1-\alpha-\beta-\alpha^{2}}\right)^{1 / \alpha}<1 .
$$

Due to (30), we get

$$
\alpha^{2} \widetilde{c}_{*} \geq\left(\widetilde{b}^{*} m_{0}^{1-\alpha^{2}}\right)^{(1 / \alpha)-1}\left(\widetilde{b}^{*} m_{0}^{1-\alpha^{2}}\right)=\left(\widetilde{b}^{*} m_{0}^{1-\alpha^{2}}\right)^{1 / \alpha}
$$

which implies

$$
m_{0} \leq\left(\frac{\alpha^{2} \widetilde{c}_{*}}{\widetilde{b}^{*(1 / \alpha)}}\right)^{\alpha /\left(1-\alpha^{2}\right)}
$$


We also obtain by (30)

$$
\begin{aligned}
\alpha^{2} \widetilde{c}_{*} \geq & \left(\widetilde{b}^{*} m_{0}^{1-\alpha^{2}}+\widetilde{d}^{*} m_{0}^{1-\alpha-\beta-\alpha^{2}}\right)^{(1 / \alpha)-1} \\
& \times\left[(1-\alpha-\beta) \widetilde{b}^{*} m_{0}^{1-\alpha^{2}}+(1-\alpha-\beta) \widetilde{d}^{*} m_{0}^{1-\alpha-\beta-\alpha^{2}}\right] \\
= & (1-\alpha-\beta)\left(\widetilde{b}^{*} m_{0}^{1-\alpha^{2}}+\widetilde{d}^{*} m_{0}^{1-\alpha-\beta-\alpha^{2}}\right)^{1 / \alpha},
\end{aligned}
$$

which leads to

$$
\left(\widetilde{b}^{*} m_{0}^{1-\alpha^{2}}+\widetilde{d}^{*} m_{0}^{1-\alpha-\beta-\alpha^{2}}\right)^{1 / \alpha} \leq \frac{\alpha^{2} \widetilde{c}_{*}}{(1-\alpha-\beta)}
$$

Combining (35) with (37), we have

$$
\begin{aligned}
m_{0}^{\alpha+1} & \left(\widetilde{b}^{*} m_{0}^{1-\alpha^{2}}+\widetilde{d}^{*} m_{0}^{1-\alpha-\beta-\alpha^{2}}\right)^{1 / \alpha} \\
& <\left(\left(\frac{\alpha^{2} \widetilde{c}_{*}}{\widetilde{b}^{*(1 / \alpha)}}\right)^{\alpha /\left(1-\alpha^{2}\right)}\right)^{\alpha+1} \frac{\alpha^{2} \widetilde{c}_{*}}{(1-\alpha-\beta)} \\
& =\left(\frac{\alpha^{2} \widetilde{c}_{*}}{\widetilde{b}^{*(1 / \alpha)}}\right)^{\alpha /(1-\alpha)} \frac{\alpha^{2} \widetilde{c}_{*}}{(1-\alpha-\beta)} \\
& =\alpha^{2 \alpha /(1-\alpha)}\left(\frac{\widetilde{c}_{*}}{\widetilde{b}^{*}}\right)^{\alpha /(1-\alpha)} \frac{\alpha^{2}}{(1-\alpha-\beta)}<1,
\end{aligned}
$$

which completes the proof.

4.2. The Case $\widetilde{e}_{*}<0<\widetilde{e}^{*}$. In this subsection, we consider (2) and (4) in the case when $\widetilde{e}_{*}<0<\widetilde{e}^{*}$. In order to study (2) by Theorem 3 , we have to set $g(x)=x^{-\lambda}, h(x)=0$, and $x \in$ $(0,+\infty)$. Let $b, c$ be the functions in condition $\left(\mathrm{H}_{1}\right)$. For the purpose of looking for $R>0$ satisfying

$$
\widetilde{e}_{*} \geq\left(\frac{\widetilde{b}^{*}}{R-\widetilde{e}^{*}}\right)^{1 / \lambda}-\frac{\widetilde{c}_{*}}{R^{\lambda}}
$$

we introduce a function on $(0,+\infty)$ by

$$
\begin{aligned}
F(m) & :=\left(\widetilde{b}^{*} m\right)^{1 / \lambda}-\frac{\widetilde{c}_{*} m^{\lambda}}{\left(1+\widetilde{e}^{*} m\right)^{\lambda}} \\
& =m^{\lambda}\left[\widetilde{b}^{*(1 / \lambda)} m^{\left(1-\lambda^{2}\right) / \lambda}-\widetilde{c}_{*}\left(1+\widetilde{e}^{*} m\right)^{-\lambda}\right] .
\end{aligned}
$$

It suffices to find $m_{0} \in(0,+\infty)$ such that $F\left(m_{0}\right) \leq \widetilde{e}_{*}$. To this end, we compute the derivative of $F$. There holds

$F^{\prime}(m)$

$$
\begin{gathered}
=\lambda m^{\lambda-1}\left[\widetilde{b}^{*(1 / \lambda)} m^{\left(1-\lambda^{2}\right) / \lambda}-\widetilde{c}_{*}\left(1+\widetilde{e}^{*} m\right)^{-\lambda}\right] \\
+m^{\lambda}\left[\frac{1-\lambda^{2}}{\lambda} \widetilde{b}^{*(1 / \alpha)} m^{\left(\left(1-\lambda^{2}\right) / \lambda\right)-1}\right. \\
\left.+\lambda \widetilde{c}_{*} \widetilde{e}^{*}\left(1+\widetilde{e}^{*} m\right)^{-\lambda-1}\right]
\end{gathered}
$$

$$
\begin{aligned}
& =\frac{1}{\lambda} m^{\lambda-1}\left\{\lambda^{2}\left[\widetilde{b}^{*(1 / \lambda)} m^{\left(1-\lambda^{2}\right) / \lambda}-\widetilde{c}_{*}\left(1+\widetilde{e}^{*} m\right)^{-\lambda}\right]\right. \\
& +\lambda m\left[\frac{1-\lambda^{2}}{\lambda} \widetilde{b}^{*(1 / \lambda)} m^{\left(\left(1-\lambda^{2}\right) / \lambda\right)-1}\right. \\
& \left.\left.+\lambda \widetilde{c}_{*} \widetilde{e}^{*}\left(1+\widetilde{e}^{*} m\right)^{-\lambda-1}\right]\right\} \\
& =\frac{1}{\lambda} m^{\lambda-1}\left[\lambda^{2} \widetilde{b}^{*(1 / \lambda)} m^{\left(1-\lambda^{2}\right) / \lambda}-\lambda^{2} \widetilde{\mathcal{c}}_{*}\left(1+\widetilde{e}^{*} m\right)^{-\lambda}\right. \\
& +\left(1-\lambda^{2}\right) \tilde{b}^{*(1 / \lambda)} m^{\left(1-\lambda^{2}\right) / \lambda} \\
& \left.+\lambda^{2} \widetilde{c}_{*} \frac{\tilde{e}^{*} m}{1+\widetilde{e}^{*} m}\left(1+\widetilde{e}^{*} m\right)^{-\lambda}\right] \\
& =\frac{1}{\lambda} m^{\lambda-1}\left[\widetilde{b}^{*(1 / \lambda)} m^{\left(1-\lambda^{2}\right) / \lambda}-\lambda^{2} \widetilde{c}_{*} \frac{1}{1+\widetilde{e}^{*} m}\left(1+\widetilde{e}^{*} m\right)^{-\lambda}\right] \\
& =\frac{m^{\lambda-1}}{\lambda\left(1+\widetilde{e}^{*} m\right)^{\lambda+1}}\left[\widetilde{b}^{*(1 / \lambda)} m^{\left(1-\lambda^{2}\right) / \lambda}\left(1+\widetilde{e}^{*} m\right)^{\lambda+1}-\lambda^{2} \widetilde{c}_{*}\right] \text {. }
\end{aligned}
$$

Assume $0<\lambda<1$. By letting

$$
\Phi(m)=: \widetilde{b}^{*(1 / \lambda)} m^{\left(1-\lambda^{2}\right) / \lambda}\left(1+\widetilde{e}^{*} m\right)^{\lambda+1}, \quad m \in[0,+\infty),
$$

we note that $\Phi$ is increasing, with $\Phi(0)=0$, and $\lim _{m \rightarrow+\infty} \Phi(m)=+\infty$. Then the equation $\Phi(m)=\lambda^{2} \widetilde{c}_{*}$ has a unique solution $m_{0} \in(0,+\infty)$, which is also the unique solution of $F(m)=0$. Since $F^{\prime}(m)<0$ for $0<m<m_{0}$ and $F^{\prime}(m)>0$ for $m>m_{0}, F$ attains the minimum at $m=m_{0}$, which shows that we get the best choice of positive constant satisfying $F(m) \leq \widetilde{e}_{*}$.

Theorem 6. Suppose that $0<\lambda<1$ and there exist continuous and nonnegative functions $b, c$ such that $b \geq p \geq c>0$. If $\widetilde{e}_{*}<0<\widetilde{e}^{*}$ and

$$
\widetilde{e}_{*} \geq m_{0}^{\lambda}\left[\widetilde{b}^{*(1 / \lambda)} m_{0}^{\left(1-\lambda^{2}\right) / \lambda}-\widetilde{c}_{*}\left(1+\widetilde{e}^{*} m_{0}\right)^{-\lambda}\right],
$$

where $m_{0}$ is the unique positive solution of the equation

$$
\widetilde{b}^{*(1 / \lambda)} m^{\left(1-\lambda^{2}\right) / \lambda}\left(1+\widetilde{e}^{*} m\right)^{\lambda+1}=\lambda^{2} \widetilde{c}_{*},
$$

then (2) has at least one positive periodic solution. 
Proof. Let $g(x)=x^{-\alpha}, h(x)=0, x \in(0,+\infty)$. Since $b \geq p \geq c$, we have, for any $t \in[0,1]$ and any and $x \in(0,+\infty), c(t) g(x) \leq p(t) g(x) \leq b(t) g(x)$. Hence, we conclude that condition $\left(\mathrm{H}_{1}\right)$ in Theorem 3 is satisfied. Taking $R=\left(1 / m_{0}\right)+\tilde{e}^{*}$, we obtain by inequality $(43)$

$$
\begin{aligned}
\widetilde{e}_{*} & \geq m_{0}^{\lambda}\left[\widetilde{b}^{*(1 / \lambda)} m_{0}^{\left(1-\lambda^{2}\right) / \lambda}-\widetilde{c}_{*}\left(1+\widetilde{e}^{*} m_{0}\right)^{-\lambda}\right] \\
& =\left(\widetilde{b}^{*} m_{0}\right)^{1 / \lambda}-\frac{\widetilde{c}_{*} m_{0}^{\lambda}}{\left(1+\widetilde{e}^{*} m_{0}\right)^{\lambda}}=\left(\frac{\widetilde{b}^{*}}{R-\widetilde{e}^{*}}\right)^{1 / \lambda}-\frac{\widetilde{c}_{*}}{R^{\lambda}} .
\end{aligned}
$$

It suffices to verify

$$
R>R^{-\lambda} \widetilde{b}^{*}+\widetilde{e}^{*}
$$

which is equivalent to

$$
\widetilde{b}^{*(1 / \lambda)} m_{0}^{1+(1 / \lambda)}<1+\widetilde{e}^{*} m_{0}
$$

It follows from (44) that $\widetilde{b}^{*(1 / \lambda)} m_{0}^{\left(1-\lambda^{2}\right) / \lambda} \leq \lambda^{2} \widetilde{c}_{*}$, which implies

$$
m_{0} \leq\left(\frac{\lambda^{2} \widetilde{c}_{*}}{\widetilde{b}^{*(1 / \lambda)}}\right)^{\lambda /\left(1-\lambda^{2}\right)}
$$

Together with the assumption that $b \geq c$, the above inequality shows

$$
\begin{aligned}
\widetilde{b}^{*(1 / \lambda)} m_{0}^{1+(1 / \lambda)} & \leq \widetilde{b}^{*(1 / \lambda)}\left(\left(\frac{\lambda^{2} \widetilde{c}_{*}}{\widetilde{b}^{*(1 / \lambda)}}\right)^{\lambda /\left(1-\lambda^{2}\right)}\right)^{(1+\lambda) / \lambda} \\
& =\widetilde{b}^{*(1 / \lambda)}\left(\frac{\lambda^{2} \widetilde{c}_{*}}{\widetilde{b}^{*(1 / \lambda)}}\right)^{1 /(1-\lambda)} \\
& =\lambda^{2 /(1-\lambda)}\left(\frac{\widetilde{c}_{*}}{\widetilde{b}^{*}}\right)^{1 /(1-\lambda)}<1<1+\widetilde{e}^{*} m_{0} .
\end{aligned}
$$

The proof is finished.

In the following, we consider (2). We also let $g(x)=x^{-\alpha}$, $h(x)=x^{\beta}$, and $x \in(0,+\infty)$. We will deal with this case in a similar manner. Let $b, c, d$ be the functions in condition $\left(\mathrm{H}_{1}\right)$. For this purpose, we note that looking for $R>0$ satisfying

$$
\widetilde{e}_{*} \geq\left(\frac{\widetilde{b}^{*}+\widetilde{d}^{*} R^{\alpha+\beta}}{R-\widetilde{e}^{*}}\right)^{1 / \alpha}-\frac{\widetilde{c}_{*}}{R^{\alpha}}
$$

is equivalent to finding $m=1 /\left(R-\widetilde{e}^{*}\right)>0$ such that

$$
\widetilde{e}_{*} \geq\left[m\left(\widetilde{b}^{*}+\widetilde{d}^{*}\left(\frac{1+\widetilde{e}^{*} m}{m}\right)^{\alpha+\beta}\right)\right]^{1 / \alpha}-\frac{\widetilde{c}_{*} m^{\alpha}}{\left(1+\widetilde{e}^{*} m\right)^{\alpha}}
$$

Based upon this observation, we have to define

$$
\begin{gathered}
F(m):=\left[m\left(\widetilde{b}^{*}+\widetilde{d}^{*}\left(\frac{1+\widetilde{e}^{*} m}{m}\right)^{\alpha+\beta}\right)\right]^{1 / \alpha}-\frac{\widetilde{c}_{*} m^{\alpha}}{\left(1+\widetilde{e}^{*} m\right)^{\alpha}} \\
=m^{\alpha}\left[\left(\widetilde{b}^{*} m^{1-\alpha^{2}}+\widetilde{d}^{*} m^{1-\alpha-\beta-\alpha^{2}}\left(1+\widetilde{e}^{*} m\right)^{\alpha+\beta}\right)^{1 / \alpha}\right. \\
\left.-\widetilde{c}_{*}\left(1+\widetilde{e}^{*} m\right)^{-\alpha}\right], \quad m \in(0,+\infty) .
\end{gathered}
$$

The derivative of $F$ can be calculated as follows:

$$
\begin{aligned}
& F^{\prime}(m) \\
& =\alpha m^{\alpha-1}\left[\left(\widetilde{b}^{*} m^{1-\alpha^{2}}+\widetilde{d}^{*} m^{1-\alpha-\beta-\alpha^{2}}\right.\right. \\
& \left.\times\left(1+\tilde{e}^{*} m\right)^{\alpha+\beta}\right)^{1 / \alpha} \\
& \left.-\widetilde{c}_{*}\left(1+\widetilde{e}^{*} m\right)^{-\alpha}\right] \\
& +m^{\alpha}\left[\frac { 1 } { \alpha } \left(\widetilde{b}^{*} m^{1-\alpha^{2}}+\widetilde{d}^{*} m^{1-\alpha-\beta-\alpha^{2}}\right.\right. \\
& \left.\times\left(1+\widetilde{e}^{*} m\right)^{\alpha+\beta}\right)^{(1 / \alpha)-1} \\
& \times\left(\left(1-\alpha^{2}\right) \tilde{b}^{*} m^{-\alpha^{2}}\right. \\
& +(\alpha+\beta) \widetilde{d}^{*} \widetilde{e}^{*} m^{1-\alpha-\beta-\alpha^{2}} \\
& \times\left(1+\widetilde{e}^{*} m\right)^{\alpha+\beta-1} \\
& +\left(1-\alpha-\beta-\alpha^{2}\right) \widetilde{d}^{*} m^{-\alpha-\beta-\alpha^{2}} \\
& \left.\times\left(1+\widetilde{e}^{*} m\right)^{\alpha+\beta}\right)
\end{aligned}
$$

$$
\left.+\alpha \widetilde{c_{*}} \widetilde{e}^{*}\left(1+\widetilde{e}^{*} m\right)^{-\alpha-1}\right]
$$

$$
\begin{aligned}
&=\frac{1}{\alpha} m^{\alpha-1} \\
& \times\left\{\alpha ^ { 2 } \left[\left(\widetilde{b}^{*} m^{1-\alpha^{2}}+\widetilde{d}^{*} m^{1-\alpha-\beta-\alpha^{2}}\right.\right.\right. \\
&\left.\quad \times\left(1+\widetilde{e}^{*} m\right)^{\alpha+\beta}\right)^{1 / \alpha} \\
&\left.-\widetilde{c}_{*}\left(1+\widetilde{e}^{*} m\right)^{-\alpha}\right] \\
&+m\left[\left(\widetilde{b}^{*} m^{1-\alpha^{2}}\right.\right. \\
&\left.\quad+\widetilde{d}^{*} m^{1-\alpha-\beta-\alpha^{2}}\left(1+\widetilde{e}^{*} m\right)^{\alpha+\beta}\right)^{(1 / \alpha)-1}
\end{aligned}
$$




$$
\begin{aligned}
& \times\left(\left(1-\alpha^{2}\right) \widetilde{b}^{*} m^{-\alpha^{2}}\right. \\
& \begin{array}{l}
+(\alpha+\beta) \widetilde{d}^{*} \widetilde{e}^{*} \frac{m}{1+\widetilde{e}^{*} m} m^{1-\alpha-\beta-\alpha^{2}} \\
\times\left(1+\widetilde{e}^{*} m\right)^{\alpha+\beta} \\
+\left(1-\alpha-\beta-\alpha^{2}\right) \widetilde{d}^{*} m^{1-\alpha-\beta-\alpha^{2}}
\end{array} \\
& +(\alpha+\beta) \widetilde{d}^{*} \widetilde{e}^{*} m^{1-\alpha-\beta-\alpha^{2}} \\
& \begin{array}{l}
+(\alpha+\beta) \widetilde{d}^{*} \widetilde{e}^{*} \frac{m}{1+\widetilde{e}^{*} m} m^{1-\alpha-\beta-\alpha^{2}} \\
\times\left(1+\widetilde{e}^{*} m\right)^{\alpha+\beta} \\
+\left(1-\alpha-\beta-\alpha^{2}\right) \widetilde{d}^{*} m^{1-\alpha-\beta-\alpha^{2}}
\end{array} \\
& \times\left(1+\widetilde{e}^{*} m\right)^{\alpha+\beta-1} \\
& +\left(1-\alpha-\beta-\alpha^{2}\right) \widetilde{d}^{*} m^{-\alpha-\beta-\alpha^{2}} \\
& \left.\times\left(1+\widetilde{e}^{*} m\right)^{\alpha+\beta}\right) \\
& \left.\left.+\alpha^{2} \widetilde{c}_{*} \widetilde{e}^{*}\left(1+\widetilde{e}^{*} m\right)^{-\alpha-1}\right]\right\} \\
& =\frac{1}{\alpha} m^{\alpha-1} \\
& \times\left\{\alpha ^ { 2 } \left[\left(\widetilde{b}^{*} m^{1-\alpha^{2}}\right.\right.\right. \\
& \left.+\widetilde{d}^{*} m^{1-\alpha-\beta-\alpha^{2}}\left(1+\widetilde{e}^{*} m\right)^{\alpha+\beta}\right)^{1 / \alpha} \\
& \left.-\widetilde{c}_{*}\left(1+\widetilde{e}^{*} m\right)^{-\alpha}\right] \\
& +\left[\left(\widetilde{b}^{*} m^{1-\alpha^{2}}+\widetilde{d}^{*} m^{1-\alpha-\beta-\alpha^{2}}\right.\right. \\
& \left.\times\left(1+\widetilde{e}^{*} m\right)^{\alpha+\beta}\right)^{(1 / \alpha)-1} \\
& \times\left(\left(1-\alpha^{2}\right) \widetilde{b}^{*} m^{1-\alpha^{2}}\right. \\
& +(\alpha+\beta) \widetilde{d}^{*} \tilde{e}^{*} m m^{1-\alpha-\beta-\alpha^{2}} \\
& \times\left(1+\widetilde{e}^{*} m\right)^{\alpha+\beta-1} \\
& +\left(1-\alpha-\beta-\alpha^{2}\right) \widetilde{d}^{*} m^{1-\alpha-\beta-\alpha^{2}} \\
& \left.\times\left(1+\widetilde{e}^{*} m\right)^{\alpha+\beta}\right) \\
& \left.\left.+\alpha^{2} \widetilde{c}_{*} \widetilde{e}^{*} m\left(1+\widetilde{e}^{*} m\right)^{-\alpha-1}\right]\right\} \\
& =\frac{1}{\alpha} m^{\alpha-1} \\
& \times\left\{\alpha ^ { 2 } \left[\left(\widetilde{b}^{*} m^{1-\alpha^{2}}\right.\right.\right. \\
& \left.+\widetilde{d}^{*} m^{1-\alpha-\beta-\alpha^{2}}\left(1+\widetilde{e}^{*} m\right)^{\alpha+\beta}\right)^{1 / \alpha} \\
& \left.-\widetilde{c}_{*}\left(1+\widetilde{e}^{*} m\right)^{-\alpha}\right] \\
& +\left[\left(\widetilde{b}^{*} m^{1-\alpha^{2}}+\widetilde{d}^{*} m^{1-\alpha-\beta-\alpha^{2}}\right.\right. \\
& \left.\times\left(1+\widetilde{e}^{*} m\right)^{\alpha+\beta}\right)^{(1 / \alpha)-1} \\
& \times\left(\left(1-\alpha^{2}\right) \widetilde{b}^{*} m^{1-\alpha^{2}}\right. \\
& \left.\times\left(1+\widetilde{e}^{*} m\right)^{\alpha+\beta}\right) \\
& \left.\left.+\alpha^{2} \widetilde{c}_{*} \widetilde{e}^{*} \frac{m}{1+\widetilde{e}^{*} m}\left(1+\widetilde{e}^{*} m\right)^{-\alpha}\right]\right\} \\
& =\frac{1}{\alpha} m^{\alpha-1} \\
& \times\left\{\left[\alpha ^ { 2 } \left(\widetilde{b}^{*} m^{1-\alpha^{2}}\right.\right.\right. \\
& \left.+\widetilde{d}^{*} m^{1-\alpha-\beta-\alpha^{2}}\left(1+\widetilde{e}^{*} m\right)^{\alpha+\beta}\right)^{1 / \alpha} \\
& \left.-\alpha^{2} \widetilde{c}_{*}\left(1+\widetilde{e}^{*} m\right)^{-\alpha}\right] \\
& +\left[\left(\widetilde{b}^{*} m^{1-\alpha^{2}}+\widetilde{d}^{*} m^{1-\alpha-\beta-\alpha^{2}}\right.\right. \\
& \left.\times\left(1+\widetilde{e}^{*} m\right)^{\alpha+\beta}\right)^{(1 / \alpha)-1} \\
& \times\left(\left(1-\alpha^{2}\right) \widetilde{b}^{*} m^{1-\alpha^{2}}\right. \\
& +(\alpha+\beta) \widetilde{d}^{*} \frac{\widetilde{e}^{*} m}{1+\widetilde{e}^{*} m} m^{1-\alpha-\beta-\alpha^{2}} \\
& \times\left(1+\widetilde{e}^{*} m\right)^{\alpha+\beta} \\
& +\left(1-\alpha-\beta-\alpha^{2}\right) \widetilde{d}^{*} m^{1-\alpha-\beta-\alpha^{2}} \\
& \left.\times\left(1+\widetilde{e}^{*} m\right)^{\alpha+\beta}\right) \\
& \left.\left.+\alpha^{2} \widetilde{c}_{*} \frac{\widetilde{e}^{*} m}{1+\widetilde{e}^{*} m}\left(1+\widetilde{e}^{*} m\right)^{-\alpha}\right]\right\} \\
& =\frac{1}{\alpha} m^{\alpha-1} \\
& \times\left\{\left[\alpha ^ { 2 } \left(\widetilde{b}^{*} m^{1-\alpha^{2}}\right.\right.\right. \\
& \left.+\widetilde{d}^{*} m^{1-\alpha-\beta-\alpha^{2}}\left(1+\widetilde{e}^{*} m\right)^{\alpha+\beta}\right)^{1 / \alpha} \\
& +\left(\widetilde{b}^{*} m^{1-\alpha^{2}}+\widetilde{d}^{*} m^{1-\alpha-\beta-\alpha^{2}}\right. \\
& \left.\times\left(1+\widetilde{e}^{*} m\right)^{\alpha+\beta}\right)^{(1 / \alpha)-1} \\
& \times\left(\left(1-\alpha^{2}\right) \widetilde{b}^{*} m^{1-\alpha^{2}}\right. \\
& +(\alpha+\beta) \widetilde{d}^{*} \frac{\widetilde{e}^{*} m}{1+\widetilde{e}^{*} m} m^{1-\alpha-\beta-\alpha^{2}}
\end{aligned}
$$




$$
\begin{aligned}
& \times\left(1+\widetilde{e}^{*} m\right)^{\alpha+\beta} \\
& +\left(1-\alpha-\beta-\alpha^{2}\right) \widetilde{d}^{*} m^{1-\alpha-\beta-\alpha^{2}} \\
& \left.\left.\times\left(1+\widetilde{e}^{*} m\right)^{\alpha+\beta}\right)\right] \\
& -\left[\alpha^{2} \widetilde{c}_{*}\left(1+\widetilde{e}^{*} m\right)^{-\alpha}\right. \\
& \left.\left.-\alpha^{2} \widetilde{c}_{*} \frac{\widetilde{e}^{*} m}{1+\widetilde{e}^{*} m}\left(1+\widetilde{e}^{*} m\right)^{-\alpha}\right]\right\} \\
& =\frac{1}{\alpha} m^{\alpha-1} \\
& \times\left\{\left(\widetilde{b}^{*} m^{1-\alpha^{2}}\right.\right. \\
& \left.+\widetilde{d}^{*} m^{1-\alpha-\beta-\alpha^{2}}\left(1+\widetilde{e}^{*} m\right)^{\alpha+\beta}\right)^{(1 / \alpha)-1} \\
& \times\left[\alpha ^ { 2 } \left(\widetilde{b}^{*} m^{1-\alpha^{2}}\right.\right. \\
& \left.+\widetilde{d}^{*} m^{1-\alpha-\beta-\alpha^{2}}\left(1+\widetilde{e}^{*} m\right)^{\alpha+\beta}\right) \\
& +\left(\left(1-\alpha^{2}\right) \tilde{b}^{*} m^{1-\alpha^{2}}\right. \\
& +(\alpha+\beta) \widetilde{d}^{*} \frac{\widetilde{e}^{*} m}{1+\widetilde{e}^{*} m} m^{1-\alpha-\beta-\alpha^{2}} \\
& \times\left(1+\widetilde{e}^{*} m\right)^{\alpha+\beta} \\
& +\left(1-\alpha-\beta-\alpha^{2}\right) \widetilde{d}^{*} m^{1-\alpha-\beta-\alpha^{2}} \\
& \left.\left.\times\left(1+\tilde{e}^{*} m\right)^{\alpha+\beta}\right)\right] \\
& -\left[\alpha^{2} \widetilde{c}_{*}\left(1+\widetilde{e}^{*} m\right)^{-\alpha}\right. \\
& \left.\left.\times\left(1-\frac{\tilde{e}^{*} m}{1+\widetilde{e}^{*} m}\right)\right]\right\} \\
& =\frac{1}{\alpha} m^{\alpha-1} \\
& \times\left\{\left(\widetilde{b}^{*} m^{1-\alpha^{2}}+\widetilde{d}^{*} m^{1-\alpha-\beta-\alpha^{2}}\right.\right. \\
& \left.\times\left(1+\widetilde{e}^{*} m\right)^{\alpha+\beta}\right)^{(1 / \alpha)-1} \\
& \times\left[\left(\alpha^{2} \widetilde{b}^{*} m^{1-\alpha^{2}}\right.\right. \\
& \left.+\left(1-\alpha^{2}\right) \tilde{b}^{*} m^{1-\alpha^{2}}\right) \\
& +\left(\alpha^{2} \widetilde{d}^{*} m^{1-\alpha-\beta-\alpha^{2}}\left(1+\widetilde{e}^{*} m\right)^{\alpha+\beta}\right.
\end{aligned}
$$

$$
\begin{aligned}
& +(\alpha+\beta) \widetilde{d}^{*} \frac{\widetilde{e}^{*} m}{1+\widetilde{e}^{*} m} m^{1-\alpha-\beta-\alpha^{2}} \\
& \times\left(1+\widetilde{e}^{*} m\right)^{\alpha+\beta} \\
& +\left(1-\alpha-\beta-\alpha^{2}\right) \tilde{d}^{*} m^{1-\alpha-\beta-\alpha^{2}} \\
& \left.\left.\times\left(1+\widetilde{e}^{*} m\right)^{\alpha+\beta}\right)\right] \\
& \left.-\alpha^{2} \widetilde{\mathcal{c}}_{*}\left(1+\widetilde{e}^{*} m\right)^{-\alpha} \frac{1}{1+\widetilde{e}^{*} m}\right\} \\
& =\frac{1}{\alpha} m^{\alpha-1} \\
& \times\left\{\left(\tilde{b}^{*} m^{1-\alpha^{2}}+\widetilde{d}^{*} m^{1-\alpha-\beta-\alpha^{2}}\right.\right. \\
& \left.\times\left(1+\widetilde{e}^{*} m\right)^{\alpha+\beta}\right)^{(1 / \alpha)-1} \\
& \times\left[\widetilde{b}^{*} m^{1-\alpha^{2}}\right. \\
& +\left(\alpha^{2}+(\alpha+\beta) \frac{\widetilde{e}^{*} m}{1+\widetilde{e}^{*} m}\right. \\
& \left.+\left(1-\alpha-\beta-\alpha^{2}\right)\right) \widetilde{d}^{*} m^{1-\alpha-\beta-\alpha^{2}} \\
& \left.\times\left(1+\widetilde{e}^{*} m\right)^{\alpha+\beta}\right] \\
& \left.-\alpha^{2} \widetilde{c}_{*}\left(1+\widetilde{e}^{*} m\right)^{-\alpha} \frac{1}{1+\widetilde{e}^{*} m}\right\} \\
& =\frac{1}{\alpha} m^{\alpha-1} \\
& \times\left\{\left(\widetilde{b}^{*} m^{1-\alpha^{2}}+\widetilde{d}^{*} m^{1-\alpha-\beta-\alpha^{2}}\right.\right. \\
& \left.\times\left(1+\widetilde{e}^{*} m\right)^{\alpha+\beta}\right)^{(1 / \alpha)-1} \\
& \times\left[\widetilde{b}^{*} m^{1-\alpha^{2}}\right. \\
& +\left(1-\frac{\alpha+\beta}{1+\widetilde{e}^{*} m}\right) \widetilde{d}^{*} m^{1-\alpha-\beta-\alpha^{2}} \\
& \left.\times\left(1+\widetilde{e}^{*} m\right)^{\alpha+\beta}\right] \\
& \left.-\alpha^{2} \widetilde{c}_{*}\left(1+\widetilde{e}^{*} m\right)^{-\alpha} \frac{1}{1+\widetilde{e}^{*} m}\right\} \\
& =\frac{1}{\alpha} \frac{m^{\alpha-1}}{\left(1+\widetilde{e}^{*} m\right)^{\alpha+1}} \\
& \times\left\{\left(\widetilde{b}^{*} m^{1-\alpha^{2}}+\widetilde{d}^{*} m^{1-\alpha-\beta-\alpha^{2}}\right.\right.
\end{aligned}
$$




$$
\begin{aligned}
& \left.\times\left(1+\widetilde{e}^{*} m\right)^{\alpha+\beta}\right)^{(1 / \alpha)-1} \\
& \times\left[\widetilde{b}^{*} m^{1-\alpha^{2}} \times\left(1+\widetilde{e}^{*} m\right)^{\alpha+1}\right. \\
& +\left(1-\alpha-\beta+\widetilde{e}^{*} m\right) \widetilde{d}^{*} m^{1-\alpha-\beta-\alpha^{2}} \\
& \left.\left.\quad \times\left(1+\widetilde{e}^{*} m\right)^{2 \alpha+\beta}\right]-\alpha^{2} \widetilde{c}_{*}\right\} .
\end{aligned}
$$

Since the function

$$
\begin{aligned}
& \Phi(m) \\
& =:\left(\widetilde{b}^{*} m^{1-\alpha^{2}}+\widetilde{d}^{*} m^{1-\alpha-\beta-\alpha^{2}}\left(1+\widetilde{e}^{*} m\right)^{\alpha+\beta}\right)^{(1 / \alpha)-1} \\
& \quad \times\left[\widetilde{b}^{*} m^{1-\alpha^{2}}\left(1+\widetilde{e}^{*} m\right)^{\alpha+1}\right. \\
& \left.\quad+\left(1-\alpha-\beta+\widetilde{e}^{*} m\right) \widetilde{d}^{*} m^{1-\alpha-\beta-\alpha^{2}}\left(1+\widetilde{e}^{*} m\right)^{2 \alpha+\beta}\right]
\end{aligned}
$$

is increasing on $m \in[0,+\infty)$ and $\Phi(0)=0$, and $\lim _{m \rightarrow+\infty} \Phi(m)=+\infty$, the equation $\Phi(m)=\alpha^{2} \bar{c}_{*}$ has a unique solution $m_{0} \in(0,+\infty)$. Thus the function $F$ attains a minimum at $m=m_{0}$, which provides a suitable $m$ satisfying (51).

Theorem 7. Suppose that $0<\alpha, \beta<1,1-\alpha-\alpha^{2}-\beta>0$, and there exist continuous and nonnegative functions $b, c, d$ on $[0,1]$ such that $b \geq c>0$ and

$$
\begin{array}{r}
c(t) x^{-\alpha} \leq p(t) x^{-\alpha}+q(t) x^{\beta} \leq b(t) x^{-\alpha}+d(t) x^{\beta}, \\
t \in[0,1], x \in(0,+\infty) .
\end{array}
$$

If $\widetilde{e}_{*}<0<\widetilde{e}^{*}$ and

$$
\begin{gathered}
\tilde{e}_{*} \geq m_{0}^{\alpha}\left[\left(\widetilde{b}^{*} m_{0}^{1-\alpha^{2}}+\widetilde{d}^{*} m_{0}^{1-\alpha-\beta-\alpha^{2}}\left(1+\widetilde{e}^{*} m_{0}\right)^{\alpha+\beta}\right)^{1 / \alpha}\right. \\
\left.-\widetilde{c}_{*}\left(1+\widetilde{e}^{*} m_{0}\right)^{-\alpha}\right]
\end{gathered}
$$

where $m_{0}$ is the unique positive solution of the equation

$$
\begin{aligned}
& \left(\widetilde{b}^{*} m^{1-\alpha^{2}}+\widetilde{d}^{*} m^{1-\alpha-\beta-\alpha^{2}}\left(1+\widetilde{e}^{*} m\right)^{\alpha+\beta}\right)^{(1 / \alpha)-1} \\
& \quad \times\left[\widetilde{b}^{*} m^{1-\alpha^{2}}\left(1+\widetilde{e}^{*} m\right)^{\alpha+1}\right. \\
& \left.\quad+\left(1-\alpha-\beta+\widetilde{e}^{*} m\right) \widetilde{d}^{*} m^{1-\alpha-\beta-\alpha^{2}}\left(1+\widetilde{e}^{*} m\right)^{2 \alpha+\beta}\right] \\
& \quad \alpha^{2} \widetilde{c}_{*},
\end{aligned}
$$

then (4) has at least one positive periodic solution.
Proof. By similar discussion in Theorem 5, we have that condition $\left(\mathrm{H}_{1}\right)$ of Theorem 3 is satisfied under the assumption in this theorem. Set $R=(1 / m)+\widetilde{e}^{*}$. Assumption (56) shows

$$
\begin{aligned}
\tilde{e}_{*} \geq & {\left[m\left(\tilde{b}^{*}+\widetilde{d}^{*}\left(\frac{1+\widetilde{e}^{*} m}{m}\right)^{\alpha+\beta}\right)\right]^{1 / \alpha} } \\
& -\frac{\widetilde{c}_{*} m^{\alpha}}{\left(1+\widetilde{e}^{*} m\right)^{\alpha}}=\left(\frac{\widetilde{b}^{*}+\widetilde{d}^{*} R^{\alpha+\beta}}{R-\widetilde{e}^{*}}\right)^{1 / \alpha}-\frac{\widetilde{c}_{*}}{R^{\alpha}} .
\end{aligned}
$$

To verify that $R$ satisfies

$$
R>R^{-\alpha}\left(\widetilde{b}^{*}+R^{\alpha+\beta} \widetilde{d}^{*}\right)+\widetilde{e}^{*},
$$

we note that, by (57), there holds

$$
\alpha^{2} \widetilde{c}_{*} \geq\left(\widetilde{b}^{*} m_{0}^{1-\alpha^{2}}\right)^{(1 / \alpha)-1}\left(\widetilde{b}^{*} m_{0}^{1-\alpha^{2}}\right)=\left(\widetilde{b}^{*} m_{0}^{1-\alpha^{2}}\right)^{1 / \alpha},
$$

which leads to

$$
m_{0} \leq\left(\frac{\alpha^{2} \widetilde{c}_{*}}{\widetilde{b}^{*(1 / \alpha)}}\right)^{\alpha /\left(1-\alpha^{2}\right)}
$$

Furthermore, we also have

$$
\begin{aligned}
\alpha^{2} \widetilde{c}_{*} \geq & \left(\widetilde{b}^{*} m_{0}^{1-\alpha^{2}}+\widetilde{d}^{*} m_{0}^{1-\alpha-\beta-\alpha^{2}}\left(1+\widetilde{e}^{*} m_{0}\right)^{\alpha+\beta}\right)^{(1 / \alpha)-1} \\
& \times\left[(1-\alpha-\beta) \widetilde{b}^{*} m_{0}^{1-\alpha^{2}}\right. \\
& \left.\quad+(1-\alpha-\beta) \widetilde{d}^{*} m_{0}^{1-\alpha-\beta-\alpha^{2}}\left(1+\widetilde{e}^{*} m_{0}\right)^{\alpha+\beta}\right] \\
= & (1-\alpha-\beta) \\
& \times\left(\widetilde{b}^{*} m_{0}^{1-\alpha^{2}}+\widetilde{d}^{*} m_{0}^{1-\alpha-\beta-\alpha^{2}}\left(1+\widetilde{e}^{*} m_{0}\right)^{\alpha+\beta}\right)^{1 / \alpha} ;
\end{aligned}
$$

that is,

$$
\frac{\alpha^{2} \widetilde{c}_{*}}{(1-\alpha-\beta)} \geq\left(\widetilde{b}^{*} m_{0}^{1-\alpha^{2}}+\widetilde{d}^{*} m_{0}^{1-\alpha-\beta-\alpha^{2}}\left(1+\widetilde{e}^{*} m_{0}\right)^{\alpha+\beta}\right)^{1 / \alpha} .
$$

Combining (61) with (63), we get

$$
\begin{aligned}
m_{0}^{\alpha+1} & \left(\widetilde{b}^{*} m_{0}^{1-\alpha^{2}}+\widetilde{d}^{*} m_{0}^{1-\alpha-\beta-\alpha^{2}}\left(1+\widetilde{e}^{*} m_{0}\right)^{\alpha+\beta}\right)^{1 / \alpha} \\
& <\left(\left(\frac{\alpha^{2} \widetilde{c}_{*}}{\widetilde{b}^{*(1 / \alpha)}}\right)^{\alpha /\left(1-\alpha^{2}\right)}\right)^{\alpha+1} \frac{\alpha^{2} \widetilde{c}_{*}}{(1-\alpha-\beta)} \\
& =\left(\frac{\alpha^{2} \widetilde{c}_{*}}{\widetilde{b}^{*(1 / \alpha)}}\right)^{\alpha /(1-\alpha)} \frac{\alpha^{2} \widetilde{c}_{*}}{(1-\alpha-\beta)} \\
& =\alpha^{2 \alpha /(1-\alpha)}\left(\frac{\widetilde{c}_{*}}{\widetilde{b}^{*}}\right)^{\alpha /(1-\alpha)} \frac{\alpha^{2}}{(1-\alpha-\beta)}<1<1+\widetilde{e}^{*} m_{0} .
\end{aligned}
$$

Substituting $R=(1 / m)+\widetilde{e}^{*}$ into the above inequality, we get (59), which completes the proof. 


\section{Example}

In this section, we apply the results established in this paper to two second-order singular equations.

Example 1. Consider the periodic problem for a second-order singular equation

$$
x^{\prime \prime}+x=\frac{\sin ^{2}(2 \pi t x)+1}{x^{1 / 2}}+x^{1 / 8}-0.03 .
$$

We will make use of Theorem 5 to show that this equation has at least one positive solution. We first note that (65) is nonresonant and the associated Green function $G$ is nonnegative. In fact, we have

$G(t, s)$

$$
=\frac{1}{2(1-\cos 1)} \begin{cases}\sin (t-s)+\sin (1-t+s), & 0 \leq s \leq t \leq 1 \\ \sin (s-t)+\sin (1-s+t), & 0 \leq t \leq s \leq 1 .\end{cases}
$$

Furthermore, there also holds $\int_{0}^{1} G(t, s) d s=1$. In order to verify the conditions in Theorem 5 , we set for any $t \in[0,1]$ and any $x \in(0,+\infty)$

$$
\begin{aligned}
a(t) & =1, \quad e(t)=-0.03 \\
f(t, x) & =\frac{\sin ^{2}(2 \pi t x)+1}{x^{1 / 2}}+x^{1 / 8} \\
b(t)=2, \quad c(t) & =d(t)=1, \quad \alpha=\frac{1}{2}, \quad \beta=\frac{1}{8} .
\end{aligned}
$$

It is easy to see that there hold $1-\alpha-\alpha^{2}-\beta=(1 / 8)>0$ and, for any $t \in[0,1]$ and $x \in(0,+\infty)$,

$$
c(t) x^{-\alpha}<f(t, x)<b(t) x^{-\alpha}+d(t) x^{\beta} .
$$

To verify (29), we point out that

$$
\begin{gathered}
\tilde{c}(t)=\widetilde{d}(t)=\frac{1}{2} \tilde{b}(t)=\int_{0}^{1} G(t, s) d s=1, \\
\widetilde{e}(t)=\int_{0}^{1} G(t, s) e(s) d s=-0.03,
\end{gathered}
$$

which lead to $\widetilde{b}^{*}=2, \widetilde{d}^{*}=\widetilde{c}_{*}=1$, and $\widetilde{e}_{*}=\widetilde{e}^{*}=-0.03$. By numerical calculation, we get the unique positive solution of equation

$$
\left(2 m^{3 / 4}+m^{1 / 8}\right)\left(2 m^{3 / 4}+\frac{3}{8} m^{1 / 8}\right)=\frac{1}{4}
$$

as $m_{0} \doteq 0.02123$. Hence, we have the desired inequalities

$$
\begin{aligned}
m_{0}^{\alpha} & {\left[\left(\tilde{b}^{*} m_{0}^{1-\alpha^{2}}+\tilde{d}^{*} m_{0}^{1-\alpha-\beta-\alpha^{2}}\right)^{1 / \alpha}-\widetilde{c}_{*}\right] } \\
& =m_{0}^{1 / 2}\left(2 m_{0}^{3 / 4}+m_{0}^{1 / 8}\right) \doteq-0.03948<\widetilde{e}_{*}<0 .
\end{aligned}
$$

Since all the conditions are satisfied, Theorem 5 guarantees that (65) has a positive solution $x$ (see Figure 1, numerical simulation for $e(t)=-0.03)$.

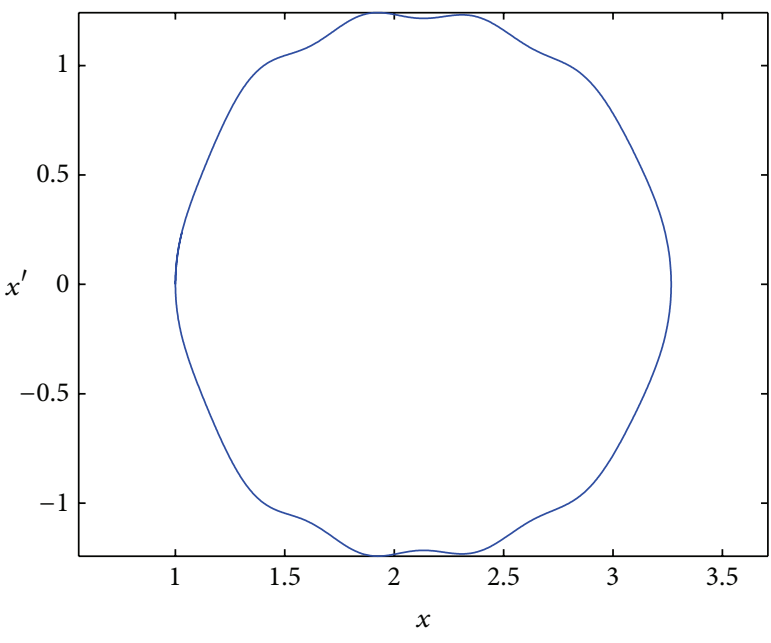

Figure 1: Numerical simulation for $e(t)=-0.03$.

Example 2. Consider the periodic problem for a secondorder singular equation

$$
\begin{aligned}
x^{\prime \prime}+x= & \frac{\sin ^{2}(2 \pi(t+x))+1}{x^{1 / 2}}+x^{1 / 8} \\
& +0.03(\sin (2 \pi t)-\max \{p(t)\}),
\end{aligned}
$$

where

$$
\begin{aligned}
p(t)= & \int_{0}^{1} G(t, s) \sin (2 \pi s) d s=(1-\cos 1)^{-1}\left(4 \pi^{2}-1\right)^{-1} \\
& \quad \times[2 \pi(1-\cos (2 \pi t) \cos (1-t)) \sin t \\
& \quad+\sin (2 \pi t)(\cos (1-t) \cos t-1)]
\end{aligned}
$$

In this example, we note that there hold

$$
\begin{gathered}
a(t)=1, \quad e(t)=0.03(\sin (2 \pi t)-\max \{p(t)\}), \\
f(t, x)=\frac{\sin ^{2}(2 \pi(t+x))+1}{x^{1 / 2}}+x^{1 / 8}, \\
t \in[0,1], x \in(0,+\infty) .
\end{gathered}
$$

To meet the conditions in Theorem 7 , we also set for any $t \epsilon$ $[0,1]$ and any $x \in(0,+\infty)$

$$
b(t)=2, \quad c(t)=d(t)=1, \quad \alpha=\frac{1}{2}, \quad \beta=\frac{1}{8} .
$$

It is clear that there hold $1-\alpha-\alpha^{2}-\beta=(1 / 8)>0$ and, for any $t \in[0,1]$ and $x \in(0,+\infty)$,

$$
c(t) x^{-\alpha}<f(t, x)<b(t) x^{-\alpha}+d(t) x^{\beta} .
$$

By direct calculation, we have $\widetilde{b}^{*}=2, \widetilde{d}^{*}=\widetilde{c}_{*}=1$, and

$$
-\widetilde{e}_{*}=\widetilde{e}^{*}=0.015 \max \{p(t)\} \doteq 0.0053706,
$$




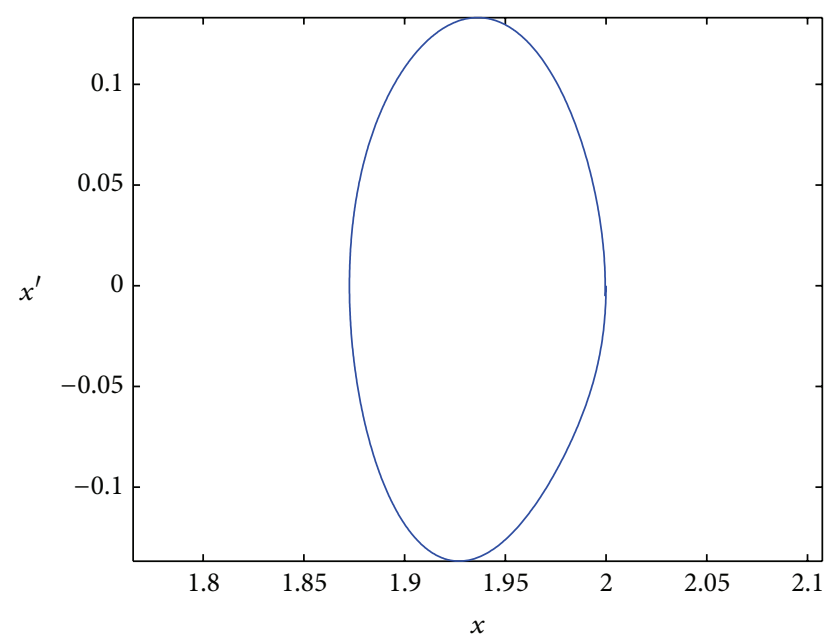

Figure 2: Numerical simulation for $e(t)=0.03 \times(\sin (2 \pi t)-$ $0.179025044320086)$.

which leads us to consider the equation

$$
\begin{aligned}
& \left(2 m^{3 / 4}+m^{1 / 8}\left(1+\widetilde{e}^{*} m\right)^{9 / 8}\right) \\
& \quad \times\left(2 m^{3 / 4}\left(1+\widetilde{e}^{*} m\right)^{\alpha+1}+\left(\frac{3}{8}+\widetilde{e}^{*} m\right) m^{1 / 8}\left(1+\widetilde{e}^{*} m\right)^{9 / 8}\right) \\
& =\frac{1}{4} .
\end{aligned}
$$

By numerical calculation, we get the unique positive solution of the above equation as $m_{0}=0.017206$. Thus, we get

$$
\begin{gathered}
m_{0}^{\alpha}\left[\left(\widetilde{b}^{*} m_{0}^{1-\alpha^{2}}+\widetilde{d}^{*} m_{0}^{1-\alpha-\beta-\alpha^{2}}\left(1+\widetilde{e}^{*} m_{0}\right)^{\alpha+\beta}\right)^{1 / \alpha}\right. \\
\left.-\widetilde{c}_{*}\left(1+\widetilde{e}^{*} m_{0}\right)^{-\alpha}\right] \\
=m_{0}^{1 / 2}\left[\left(2 m_{0}^{3 / 4}+m_{0}^{1 / 8}\left(1+\widetilde{e}^{*} m_{0}\right)^{5 / 8}\right)^{2}\right. \\
\left.-\left(1+\widetilde{e}^{*} m_{0}\right)^{-1 / 2}\right] \doteq-0.06804<\widetilde{e}_{*} .
\end{gathered}
$$

Together with the fact that $\widetilde{e}_{*}<0<\widetilde{e}^{*}$, the above inequality shows that all the conditions of Theorem 7 are satisfied. Consequently, Theorem 7 guarantees that (72) has a positive solution $x$ (see Figure 2, numerical simulation).

\section{Conflict of Interests}

The authors declare that there is no conflict of interests regarding the publication of this paper.

\section{Acknowledgments}

The work was supported by Program for Changjiang Scholars and Innovative Research Team in University, NSFC of China (no. 10971021) and Key Subject of Chinese Ministry of Education (no. 109051), Scientific Research Fund of Heilongjiang Provincial Education Department (no. 11544032), a Grant from the Ph.D. Programs Foundation of Ministry of Education of China (no. 200918), and Youth Education Jun Projects of Jilin University of Finance and Economics.

\section{References}

[1] J. Chu and P. J. Torres, "Applications of Schauder's fixed point theorem to singular differential equations," Bulletin of the London Mathematical Society, vol. 39, no. 4, pp. 653-660, 2007.

[2] P. J. Torres, "Weak singularities may help periodic solutions to exist," Journal of Differential Equations, vol. 232, no. 1, pp. 277284, 2007.

[3] M. Zhang, "Periodic solutions of equations of Emarkov-Pinney type," Advanced Nonlinear Studies, vol. 6, no. 1, pp. 57-67, 2006.

[4] J. Chen and D. O’Regan, "On periodic solutions for even order differential equations," Nonlinear Analysis. Theory, Methods \& Applications, vol. 69, no. 4, pp. 1138-1144, 2008.

[5] M. A. del Pino, R. F. Manásevich, and A. Montero, “T-periodic solutions for some second order differential equations with singularities," Proceedings of the Royal Society of Edinburgh A: Mathematics, vol. 120, no. 3-4, pp. 231-243, 1992.

[6] M. A. del Pino and R. F. Manasevich, "Infinitely many Tperiodic solutions for a problem arising in nonlinear elasticity," Journal of Differential Equations, vol. 103, no. 2, pp. 260-277, 1993.

[7] A. Fonda, R. Manásevich, and F. Zanolin, "Subharmonic solutions for some second order di fferential equations with singularities," SIAM Journal on Mathematical Analysis, vol. 24, no. 5, pp. 1294-1311, 1993.

[8] D. Franco and J. R. L. Webb, "Collisionless orbits of singular and non singular dynamical systems," Discrete and Continuous Dynamical Systems. Series A, vol. 15, no. 3, pp. 747-757, 2006.

[9] W. B. Gordon, "Conservative dynamical systems involving strong forces," Transactions of the American Mathematical Society, vol. 204, pp. 113-135, 1975.

[10] P. Habets and L. Sanchez, "Periodic solutions of some Liénard equations with singularities," Proceedings of the American Mathematical Society, vol. 109, no. 4, pp. 1035-1044, 1990.

[11] D. Jiang, J. Chu, D. O’Regan, and R. P. Agarwal, “Multiple positive solutions to superlinear periodic boundary value problems with repulsive singular forces," Journal of Mathematical Analysis and Applications, vol. 286, no. 2, pp. 563-576, 2003.

[12] D. Jiang, J. Chu, and M. Zhang, "Multiplicity of positive periodic solutions to superlinear repulsive singular equations," Journal of Differential Equations, vol. 211, no. 2, pp. 282-302, 2005.

[13] A. C. Lazer and S. Solimini, "On periodic solutions of nonlinear differential equations with singularities," Proceedings of the American Mathematical Society, vol. 99, no. 1, pp. 109-114, 1987.

[14] Z. Cao and D. Jiang, "Periodic solutions of second order singular coupled systems," Nonlinear Analysis. Theory, Methods \& Applications. An International Multidisciplinary Journal A: Theory and Methods, vol. 71, no. 9, pp. 3661-3667, 2009.

[15] I. Rachůnková, M. Tvrdý, and I. Vrkoč, "Existence of nonnegative and nonpositive solutions for second order periodic boundary value problems," Journal of Differential Equations, vol. 176, no. 2, pp. 445-469, 2001.

[16] P. J. Torres, "Existence of one-signed periodic solutions of some second-order differential equations via a Krasnoselskii fixed 
point theorem," Journal of Differential Equations, vol. 190, no. 2, pp. 643-662, 2003.

[17] J. Chu, X. Lin, D. Jiang, D. O'Regan, and R. P. Agarwal, "Multiplicity of positive periodic solutions to second order differential equations," Bulletin of the Australian Mathematical Society, vol. 73, no. 2, pp. 175-182, 2006.

[18] P. Yan and M. Zhang, "Higher order non-resonance for differential equations with singularities," Mathematical Methods in the Applied Sciences, vol. 26, no. 12, pp. 1067-1074, 2003.

[19] M. Zhang, "A relationship between the periodic and the Dirichlet BVPs of singular differential equations," Proceedings of the Royal Society of Edinburgh A. Mathematics, vol. 128, no. 5, pp. 1099-1114, 1998.

[20] F. Zhu, L. Liu, and Y. Wu, "Positive solutions for systems of a nonlinear fourth-order singular semipositone boundary value problems," Applied Mathematics and Computation, vol. 216, no. 2, pp. 448-457, 2010.

[21] M. Yao, A. Zhao, and J. Yan, "Periodic boundary value problems of second-order impulsive differential equations," Nonlinear Analysis. Theory, Methods \& Applications. An International Multidisciplinary Journal A: Theory and Methods, vol. 70, no. 1, pp. 262-273, 2009. 


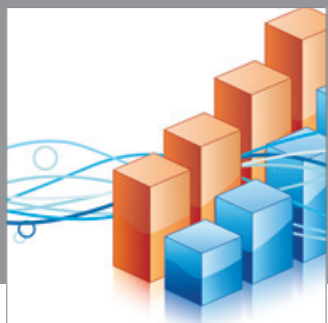

Advances in

Operations Research

mansans

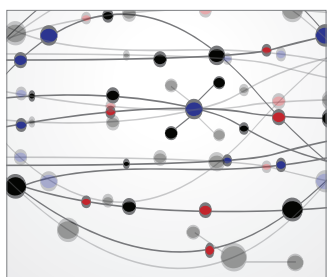

The Scientific World Journal
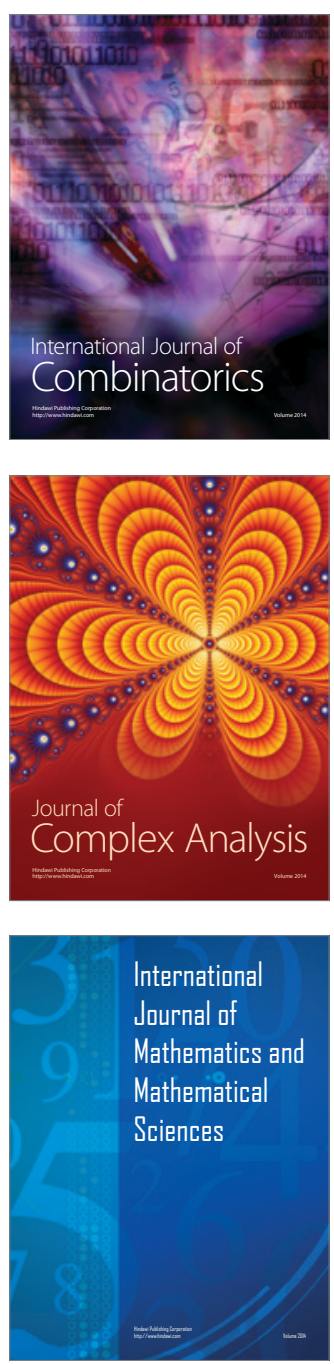
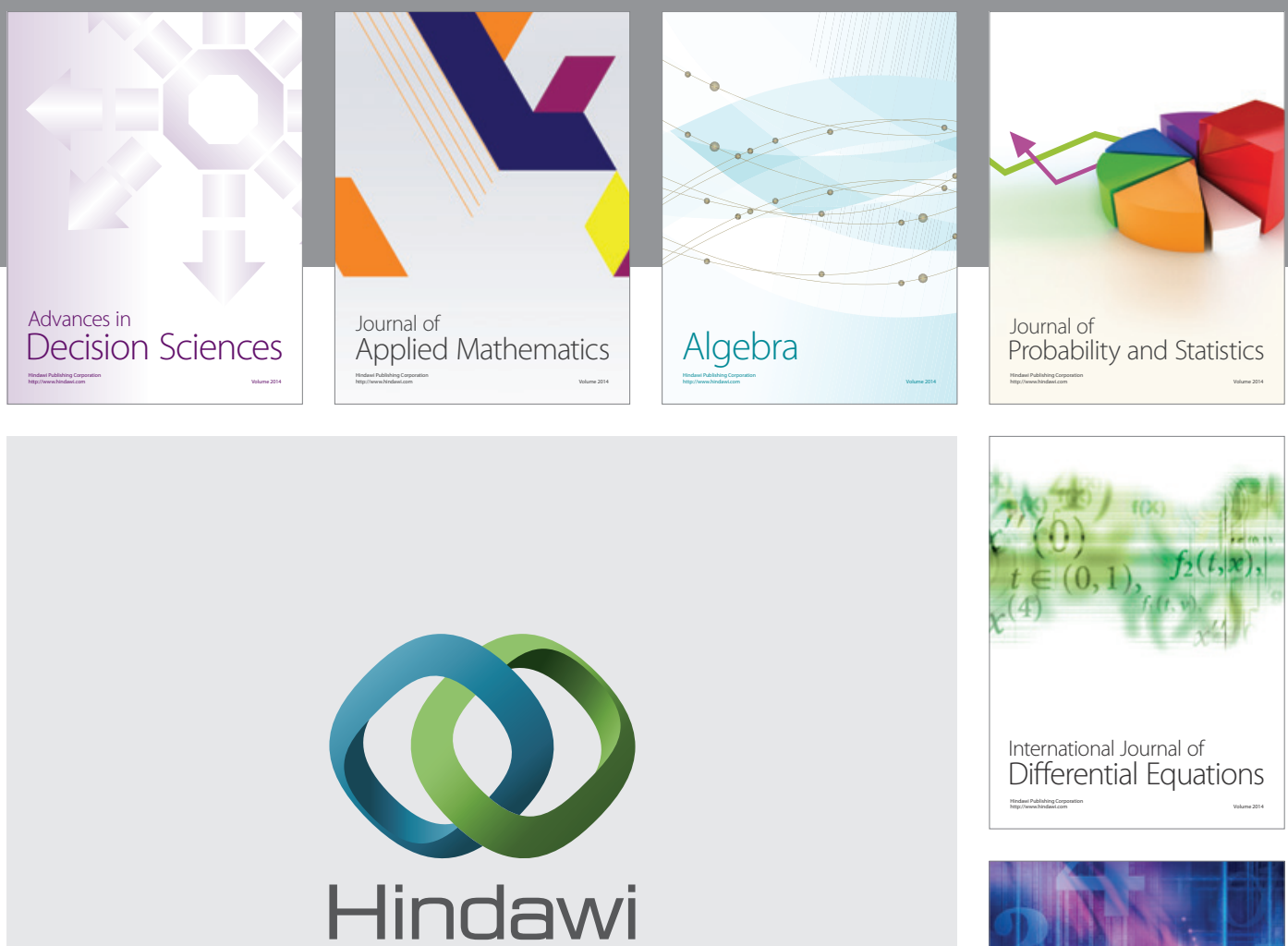

Submit your manuscripts at http://www.hindawi.com
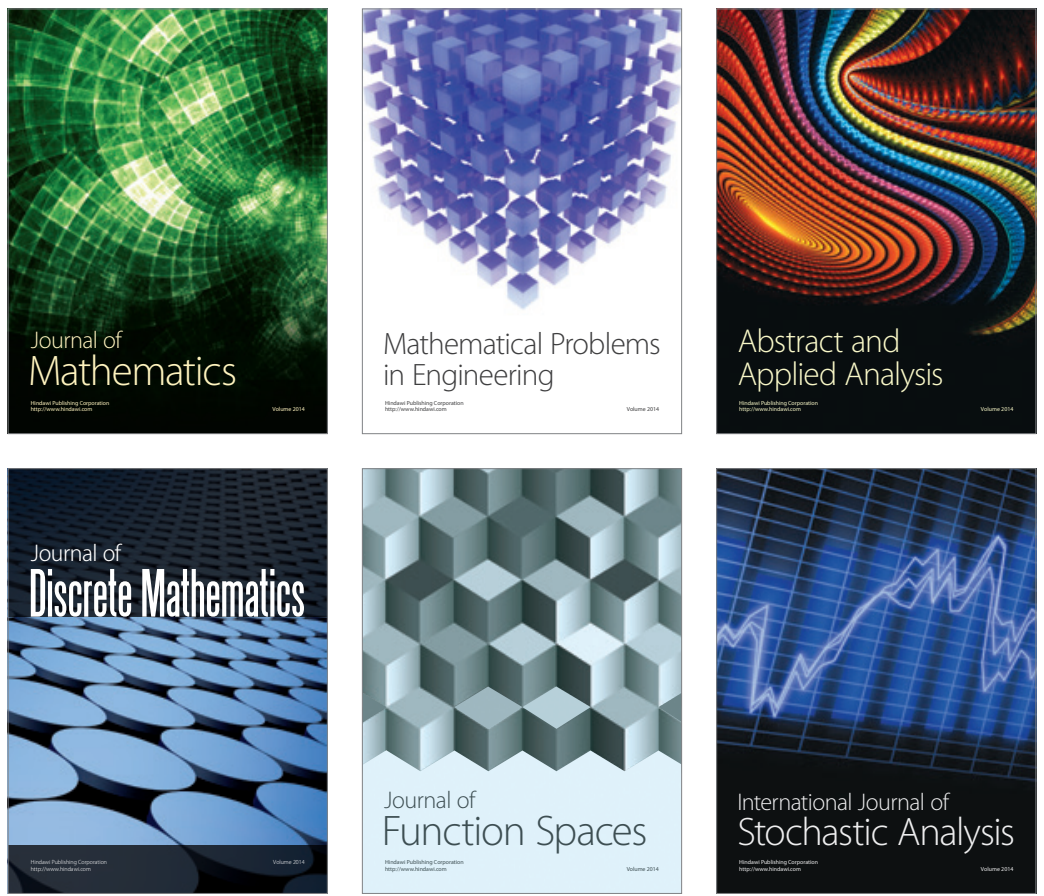

Journal of

Function Spaces

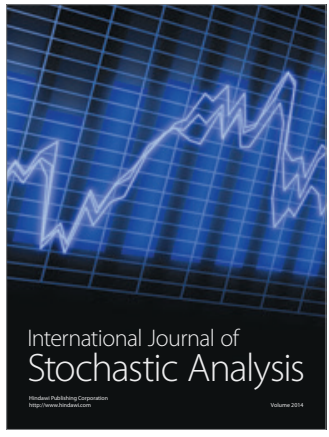

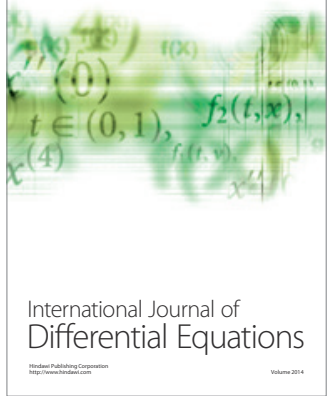
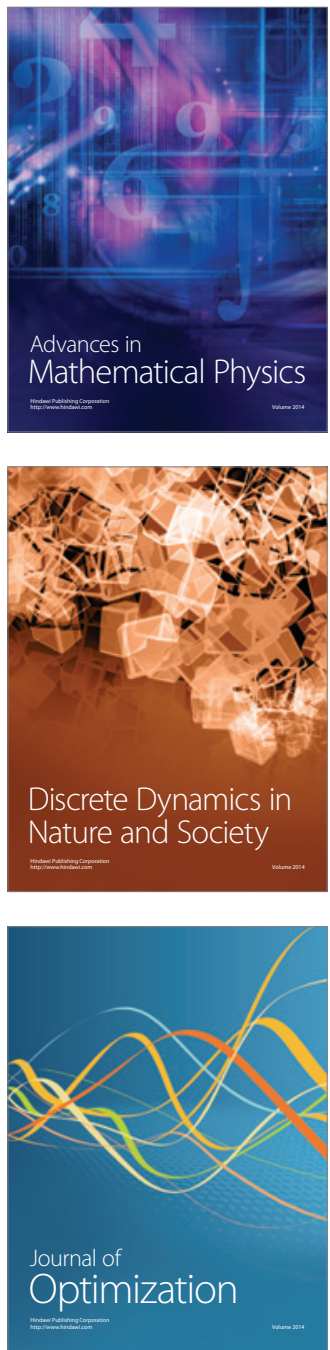